\title{
Surgical ventricular remodeling: A balancing act on systolic and diastolic properties
}

\author{
Daniel Burkhoff, MD, PhD, ${ }^{a}$ and Andrew S. Wechsler, $\mathrm{MD}^{\mathrm{b}}$
}

See related article on page 610 .

From the Cardiovascular Research Foundation, Orangeburg, NY, ${ }^{a}$ and the Department of Cardiothoracic, Surgery, Drexel University College of Medicine, Philadelphia, Pa. ${ }^{\text {b }}$

Received for publication April 26, 2006; accepted for publication April 27, 2006.

Address for reprints: Daniel Burkhoff, MD, $\mathrm{PhD}$, The Jack H Skirball Center for Cardiovascular Research, Cardiovascular Research Foundation, 8 Corporate Dr, Orangeburg, NY 10962 (E-mail: dburkhoff@ crf.org).

J Thorac Cardiovasc Surg 2006;132:459-63

$0022-5223 / \$ 32.00$

Copyright $(\odot) 2006$ by The American Association for Thoracic Surgery

doi:10.1016/j.jtcvs.2006.04.045
D ne of the fundamental characteristics of chronic heart failure is the progressive chamber dilation and deterioration of pump function that is driven by the increased hemodynamic and neurohormonal stresses present in this condition. ${ }^{1}$ This process, referred to as ventricular remodeling, involves structural, cellular, extracellular, molecular, biochemical, and metabolic mechanisms. It is now widely appreciated that remodeling is not just a manifestation of disease, but is an important mechanism of disease. Considering just the structural aspects of remodeling, it follows from Laplace's law (pressure $\propto$ wall tension/radius) that even with normal myocardial force-generating capacity, chamber pumping strength decreases as chamber radius increases. Thus, many therapies are now evaluated on their ability to restore normal heart size and induce reverse remodeling. At least some of the benefits of angiotensin-converting enzyme inhibitors, ${ }^{2} \beta$-blockers, ${ }^{3}$ cardiac resynchronization therapy, ${ }^{4}$ and passive ventricular constraint ${ }^{5}$ are attributed to their ability to inhibit or reverse remodel the heart. However, for a vast majority of patients, the degree of reverse remodeling achieved with these standard approaches is limited.

For almost 50 years, various techniques have been developed for surgically correcting the dilated left ventricle that accompanies systolic heart failure. ${ }^{6-9}$ Surgical resection or exclusion of a section of the dilated chamber reduces chamber radius and by Laplace's law has been postulated to increase ventricular pressureand flow-generating capability. Indeed, prior studies in patients with ischemic ${ }^{10}$ or idiopathic $^{11,12}$ cardiomyopathies have shown that such surgical ventricular restoration (SVR) reduces chamber size and increases ejection fraction (EF), seemingly providing physiologic evidence of improved pump function.

However, when applied to patients with idiopathic cardiomyopathy (with a form of SVR commonly referred to as the Batista operation), clinical outcomes were poor despite the fact that these apparently beneficial physiologic effects were achieved. ${ }^{11,12}$ Many surviving patients continued to experience symptoms and there was a high rate of transplantation or ventricular assist device implantation. Consequently, this procedure is no longer practiced. It is noteworthy, however, that these poor clinical outcomes were prospectively predicated (before availability of any clinical data) on the basis of theoretical considerations of the impact of resecting a portion of the idiomyopathic ventricular wall on overall pump function. ${ }^{13}$ That analysis stressed two important concepts: (1) in addition to enhancing certain aspects of systolic chamber properties, resection of a portion of the wall also can detrimentally affect diastolic chamber properties and (2) the effect of such a procedure on overall pump function reflects the balance of its effects on systolic and diastolic properties. In the case of the Batista operation, the detrimental effects on diastole exceeded the beneficial effects on systole so that the net impact on pump function was detrimental. ${ }^{13,14}$ These important concepts were subsequently confirmed in a detailed hemodynamic study of patients who underwent the Batista operation before it was abandoned. ${ }^{15}$

\section{SVR in Ischemic Cardiomyopathy}

In contrast to observations in idiopathic cardiomyopathy, when applied to patients with ischemic cardiomyopathy, clinical outcomes after SVR appear to be considerably better and more predictable. ${ }^{10}$ Unlike the situation in idiopathic cardiomyopathy, where weak 


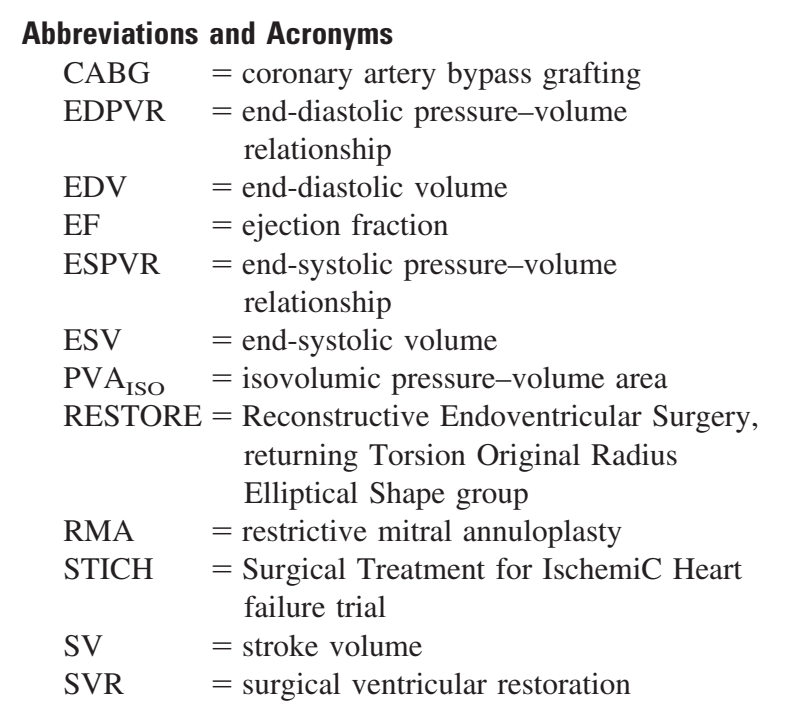

but contracting muscle is removed, the surgical target in ischemic cardiomyopathy is akinetic or dyskinetic scar. Concordant with clinical observations, theoretical analysis predicts a neutral impact (in the case of akinetic scar exclusion) or a beneficial impact (in the case of dyskinetic scar exclusion) on overall pump function. ${ }^{14}$

In this issue of The Journal of Thoracic and Cardiovascular Surgery, Tulner and associates ${ }^{16}$ report a detailed hemodynamic study of SVR in 10 patients with ischemic cardiomyopathy. Seven patients had dyskinetic scar and 3 patients had akinetic scar. In addition to SVR, all patients underwent coronary artery bypass grafting (CABG) and 7 patients also had restrictive mitral annuloplasty (RMA). Results obtained from these patients were compared with those obtained in patients who underwent CABG alone, RMA alone, or CABG plus RMA, which served as active control groups. pressure-volume relationships at end-systole and end-diastole (ESPVR and EDPVR, respectively) were measured preoperatively and postoperatively as the primary means of assessing the impact of SVR on ventricular properties in addition to standard hemodynamic measures. The ESPVR is the line connecting the end-systolic pressure and volume points generated by a series of variably loaded heartbeats. The ESPVR is relatively unaffected by loading conditions and reflects intrinsic chamber contractile properties. Similarly, the EDPVR is the line connecting the end-diastolic pressure and volumes generated from variably loaded heartbeats, and provides a means of assessing passive ventricular properties. ${ }^{17}$

Results in the CABG and RMA (including combined CABG and RMA) groups showed essentially no significant impact on the key parameters of the pressure-volume relations. These results are intended to support the contention that findings in the SVR group can be attributed primarily to SVR and not to cardiopulmonary bypass, cardioplegia, CABG, or to RMA.

In terms of basic hemodynamic parameters (Table 3 of Tulner and associates ${ }^{16}$ ), SVR resulted in lower end-diastolic (EDV) and end-systolic volumes (ESV), lower peak wall stress, and higher EF. However, stroke volume (SV; ie, EDV-ESV) tended to decrease, ${ }^{*}$ systolic blood pressure was lower, and end-diastolic pressure was significantly higher. All of these hemodynamic effects are reflected in the changes in the average pressure-volume loops (reproduced here in Figure 1, A). With regard to pressure-volume relations, there were leftward shifts of both EDPVR and ESPVR toward more normal volumes. The average results of pressure-volume analysis are illustrated in Figure 1, $B$ (plots derived from information provided in Table 4 of Tulner and associates ${ }^{16}$ ).

The fundamental question to be addressed in this editorial is whether, in aggregate, these changes in hemodynamics and ventricular properties induced by surgically reducing the size of the heart are favorable or unfavorable. pressure-volume analysis provides the most comprehensive means of assessing ventricular contractile properties, ${ }^{17}$ and the most rigorous means of measuring these relations in the clinical setting is with the conductance catheter ${ }^{18}$ as used by Tulner and colleagues. ${ }^{16}$

\section{Theoretical and Physiologic Considerations}

As discussed above, the reductions in EDV and ESV achieved by SVR in and of themselves have been purported to fundamentally yield a stronger pump. The increase in EF seemingly provides support for this assertion, although it is intuitively obvious that reduction of EDV without an increase in EF would result in inadequate SV. However, with "usual" inotropic interventions (eg, $\beta$-adrenergic agents, resolution of ischemia), an increase of EF results from an increase in SV with little change in EDV. In contrast, the increase in EF observed with SVR results from a decrease in EDV with little change in SV. Thus, as noted previously, ${ }^{13,14}$ increases in EF after SVR do not have the usual meaning and should not be interpreted as providing evidence of increased pump strength in this setting. In fact, the reduced peak and end-systolic left ventricular pressures (decreased afterload) and marked elevation of end-diastolic pressure (increased preload) with no increase in SV observed after SVR (Figure 1, A) could actually imply reduced pumping capability.

This conclusion is further supported by additional analysis of the relative shifts of pressure-volume relations as detailed in Figure 1, B. With $\mathrm{ESV}_{80}$ (the ESV at a pressure

* Changes in SV were not reported directly by Tulner and associates ${ }^{16}$ but can be estimated as the difference between mean EDV and mean ESV from data in Table 3. In this case, there is a trend for SV to decrease from 64 to $59 \mathrm{~mL}$. However, it cannot be determined from these data whether this decrease is statistically significant. 
A

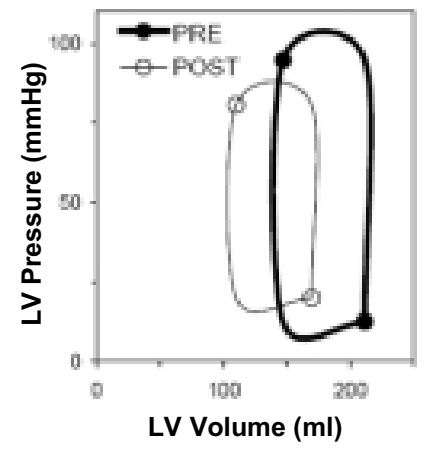

C

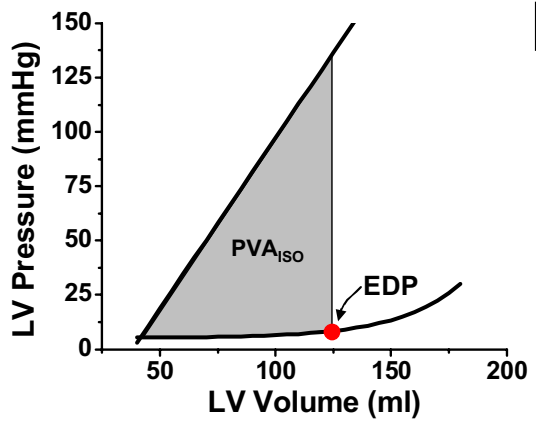

B

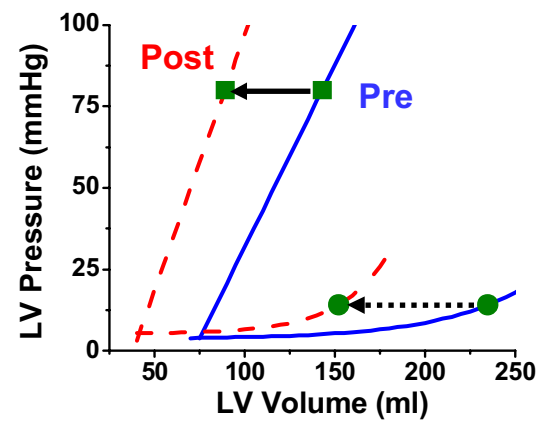

D

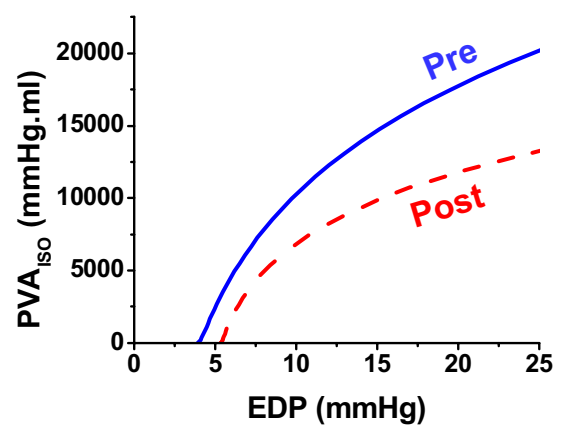

Figure 1. A, Reproduction of the average pressure-volume loops obtained by Tulner and associates. ${ }^{16}$ B, Endsystolic and end-diastolic pressure-volume relations were constructed from the average results presented in Table 4 of Tulner and associates. ${ }^{16}$ Preoperative (PRE) relations are shown by solid lines, and postoperative (POST) relations are shown by dashed lines. Volumes at a systolic pressure of $80 \mathrm{~mm} \mathrm{Hg}$ are shown by squares, and diastolic volumes at a pressure of $14 \mathrm{~mm} \mathrm{Hg}$ are shown by circles. Arrows show respective shift of ESPVR (solid lined arrow) and EDPVR (dashed line arrow), respectively. The shift of EDPVR is greater than the shift of the ESPVR. C, Demonstration of how isovolumic pressure-volume area $\left(P V A_{\text {Iso }}\right.$ ) is calculated from the pressure-volume diagram.

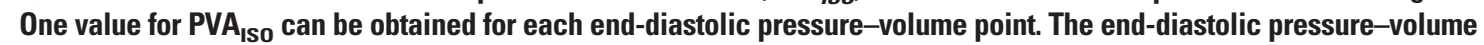
point (EDP) for this example is shown by the solid red circle. D, The relationship between PVA Iso $_{\text {and EDP is }}$ constructed from the preoperative and postoperative EDPVRs and ESPVRs of panel B. The postoperative relationship is lower than the preoperative relation, signifying a reduction in the maximum work capacity of the heart.

of $80 \mathrm{~mm} \mathrm{Hg}$, as detailed by Tulner and colleagues ${ }^{16}$ ) used to index the ESPVR position, SVR resulted in an approximately $54 \mathrm{~mL}$ leftward shift of the average ESPVR (green squares) with only a small increase in the slope of the ESPVR. This compares with a leftward EDPVR shift of approximately $83 \mathrm{~mL}$ using $\mathrm{EDV}_{14}$ (the EDV at a pressure of $14 \mathrm{~mm} \mathrm{Hg}$, green circles) to index the EDPVR position; chamber stiffness is also increased, which further accentuates the impact on diastolic properties. Thus, the magnitude of the leftward shift of the EDPVR is approximately $30 \mathrm{~mL}$ greater than that of the ESPVR.

This, in turn, signifies a reduction of overall pump function as revealed by the overall pump function curves (Figure 1,D), which show isovolumic pressure-volume area $\left(\mathrm{PVA}_{\mathrm{ISO}}\right)$ as a function of end-diastolic pressure. ${ }^{13,14} \mathrm{PVA}_{\mathrm{ISO}}$ is the area on the pressure-volume diagram of the triangular region contained between the EDPVR and ESPVR at each EDV (Figure 1,C). At increasing EDVs, end-diastolic pressure increases

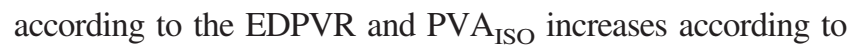
the relative positions of the EDPVR and ESPVR. PVA ISO $_{\text {is a }}$ measure of the total possible mechanical energy the ventricle can generate at the specified preload pressure and thus provides an afterload-independent measure of the pumping capability of the heart. As depicted in Figure 1, D, the relationship between

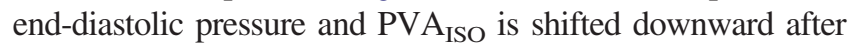
SVR, indicating that at any given filling pressure the heart is capable of less work than before the procedure. In aggregate, the changes in the pressure-volume loops and pressure-volume relationships resemble those observed in classic cases of diastolic heart failure. ${ }^{19}$ However, this interpretation must be tempered by the realization that the heart is now working at more physiologic volumes.

One additional index examined by Tulner and colleagues ${ }^{16}$ was mechanical efficiency (defined as the ratio between external stroke work and pressure-volume area), which was improved after SVR. Although it is fashionable 
to examine various measures of mechanical or metabolic efficiency, the clinical and even physiologic meaning of these measures is questionable. An "efficient" heart that does not adequately perfuse the body or requires pathologically elevated filling pressures or reduced arterial pressures to do so cannot be considered desirable. Furthermore, examination of the average pressure-volume loops (Figure 1, A) clearly shows a rather substantial reduction in stroke work (the area inside the loop) after SVR. It would have been interesting to see a comparison of the stroke work curves at more physiologic pressures after bypass.

Finally, wall stress is reduced by SVR. This has long been theorized to facilitate myocyte shortening and, in the long term, permit regression of hypertrophy (ie, reverse remodeling of the cells). Since SV is not increased, facilitation of myocyte shortening cannot be confirmed. At the present time, there are no data to confirm or refute reverse cellular remodeling, so this remains a theoretical possibility whose clinical implications would require clarification. The ultimate question that remains is to determine the balance between the relatively beneficial effects of decreasing myocardial wall stress and the detrimental effects of increasing diastolic filling pressures as a consequence of reducing chamber volume while not importantly increasing SV.

\section{Surgical and Clinical Considerations}

Several factors must be considered in determining the applicability of the results of the findings of Tulner and coworkers ${ }^{16}$ to SVR in general.

First, the physiologic measurements were made in the moments after completion of the surgical procedure and separation from cardiopulmonary bypass. Although measurements made in CABG and RMA patients showed no statistically significant impact on any of the measurements, SVR procedures were significantly longer, including longer cardiopulmonary bypass and aortic crossclamp times, and are considerably more invasive. These factors could result in myocardial stunning and/or myocardial edema (potentially contributing to diastolic stiffening), each of which could recover over time, rendering the present results limited to the postoperative period. Decreased ventricular compliance has been demonstrated in most other studies in the setting of prolonged myocardial ischemia. $^{20}$

Second, SVR is not a single operative procedure but, as reviwed by Griffith, ${ }^{9}$ is a class of operations that have evolved over almost 50 years and includes many different approaches. For ischemic cardiomyopathy the most widely practiced techniques today are variations of endoventricular circular patch plasty as described by Dor and associates, ${ }^{21}$ which was used by Tulner and colleagues. ${ }^{16}$ However, the consistency with which these procedures are performed between operators is currently unknown. Nonstandardized aspects of these procedures include the following: methods of identifying akinetic and dys- kinetic scar; methods of myocardial protection; criteria for identifying the border zone between viable and nonviable myocardium for placement of the encircling stitch; determination of the ideal size of the remodeled heart; methods for restoring normal geometry; means of customizing the patch to complete the closure or even the decision of whether to use a patch; and the extent to which simultaneous revascularization is used. Results obtained by Tulner and colleagues ${ }^{16}$ (who used a balloon adjusted to $55 \mathrm{~mL} / \mathrm{m}^{2}$ ) therefore may or may not apply to procedures performed by other surgical groups. For example, Schreuderr et al also measured pressure-volume relations following SVR of post-infarct scar. ${ }^{22}$ Based on raw data presented from 2 patients, it appears that in contrast to Tulner et al, ${ }^{16}$ the shifts of the ESPVRs and EDPVRs reported by this group are very similar to each other, which suggests that there would be no significant change in overall pump function as

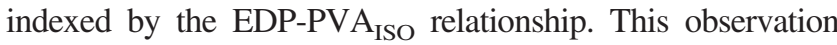
more closely parallels what we predicted for SVR of a heart with an akinetic scar ${ }^{14}$ than what was observed by Tulner et al. ${ }^{16}$ Moreover, we know from frequent presentations by Dor that excessive reduction of ventricular chamber size is associated with a low output state and restricted left ventricular filling (diastolic dysfunction).

For further comparison, the RESTORE group registry has now reported on almost 1200 patients who underwent SVR. ${ }^{23}$ CABG and/or mitral valve repair are performed in a vast majority of patients. Thirty-day mortality is reported as approximately $5 \%$, and fewer than $10 \%$ of patients require mechanical support (which was mainly in the form of intra-aortic balloon pumping). ${ }^{10,23}$ In the latest report from this registry, EF increased from $29.6 \% \pm 11.0 \%$ to $39 \% \pm 12.3 \%$ and left ventricular ESV index decreased from $80 \pm 51$ to $57 \pm 34$ $\mathrm{mL} / \mathrm{m}^{2}{ }^{23}$ These results compare favorably with the preoperative and postoperative measurements reported by Tulner and colleagues. ${ }^{16}$ Follow-up studies by the RESTORE group have shown good long-term survival $\left(89 \%\right.$ at 18 months $^{10}$ and almost $70 \%$ at 5 years ${ }^{23}$ ) and sustained clinical improvement in heart failure class. ${ }^{23}$ In the absence of additional studies, it is hard to determine whether this outcome is a result of reverse remodeling. Interestingly, such excellent outcomes are also consistent with the theoretically predicted neutral or beneficial effects on overall pump function of SVR in ischemic cardiomyopathy. ${ }^{13,14}$ Tulner and colleagues ${ }^{16}$ have not yet provided long-term follow-up of these specific patients. Such follow-up will be very interesting and is ultimately required for thoroughly understanding the meaning of the present findings.

\section{Implications}

The findings of the present study are important for several reasons. First and foremost, this study emphasizes the importance of using the pressure-volume approach for understanding the impact of SVR on ventricular pump function. Had our knowledge been restricted to what we could glean 
from measurements of volumes and EF, we would not understand the fundamental concept of the interplay between the effects on systole and diastole, which may ultimately be a key in optimizing SVR. Second, on a practical level, understanding the immediate hemodynamic effects of SVR can help establish optimized standards for postoperative care. For example, if myocardial edema and/or myocardial stunning are indeed important factors, treatment strategies specifically directed at these factors can be planned. Third, as noted by Tulner and coworkers, ${ }^{16}$ the present results emphasize the need for understanding the long-term effects of SVR on the pressure-volume relations, and ideally these studies will be performed. Fourth, these finding emphasize that understanding the acute and chronic hemodynamic effects could potentially help optimize patient selection criteria.

There is considerable controversy about the clinical utility of SVR. Many surgeons are eager to perform this procedure, are impressed by the reductions in volumes and increases of $\mathrm{EF}$, and accept these as proof that the procedure provides clinical benefit. Many physicians (surgeons and heart failure physicians alike) remain skeptical of the benefits of this major surgical procedure (with defined mortality and morbidity) and are reluctant to refer patients based solely on data provided from small surgical series and voluntary registries (no matter how large). If we learned anything from the experience with the Batista operation, we learned that surgically remodeling the dilated heart is not always beneficial. With this experience still fresh in our minds, many physicians await objective data of improved functional status, quality of life, exercise tolerance or, most important, mortality. The STICH trial, sponsored by the National Institutes of Health, is designed to address the impact of SVR on mortality. Until the results of this study are available, significant insights and procedural acceptance can be obtained from small, carefully executed physiologic studies demonstrating unequivocal hemodynamic benefit. In this regard, the study performed by Tulner and colleagues ${ }^{16}$ has been masterfully executed and has yielded very valuable data that raise several questions about the short-term hemodynamic impact of SVR. Ideally, additional information will be provided from studies performed on patients at later time points. The single most important concept derived from prior studies and confirmed in this study is that when the heart is surgically remodeled both systole and diastole are affected, and it is a complex matter to predict the impact on overall pump function.

\section{References}

1. Mancini D, Burkhoff D. Mechanical device-based methods of managing and treating heart failure. Circulation. 2005;112:438-48.

2. St John SM, Pfeffer MA, Moye L, Plappert T, Rouleau JL, Lamas G, et al. Cardiovascular death and left ventricular remodeling two years after myocardial infarction: baseline predictors and impact of longterm use of captopril-information from the Survival and Ventricular Enlargement (SAVE) trial. Circulation. 1997;96:3294-9.

3. Udelson JE. Ventricular remodeling in heart failure and the effect of beta-blockade. Am J Cardiol. 2004;93:43B-8B.
4. John Sutton MG, Plappert T, Abraham WT, Smith AL, Delurgio DB, Leon AR, et al. Effect of cardiac resynchronization therapy on left ventricular size and function in chronic heart failure. Circulation. 2003;107:1985-90.

5. Saavedra WF, Tunin RS, Paolocci N, Mishima T, Suzuki G, Emala $\mathrm{CW}$, et al. Reverse remodeling and enhanced adrenergic reserve from passive external support in experimental dilated heart failure. $J \mathrm{Am}$ Coll Cardiol. 2002;39:2069-76.

6. Lidoff W, Bailey CP. Ventriculoplasty: excision of myocardial aneurysm-report of a successful case. JAMA. 1955;158:915-20.

7. Jatene AD. Left ventricular aneurysmectom: resection or reconstruction. J Thorac Cardiovasc Surg. 1985;89:321-31.

8. Dor V, Saab M, Coste P, Kornaszewska M, Montiglio F. Left ventricular aneurysm: a new surgical approach. Thorac Cardiovasc Surg. 1989;37:11-9.

9. Griffith BP. Surgical treatment of congestive heart failure: evolving options. Ann Thorac Surg. 2003;76:S2254-9.

10. Athanasuleas CL, Stanley AW Jr, Buckberg GD, Dor V, DiDonato M, Blackstone EH. Surgical anterior ventricular endocardial restoration (SAVER) in the dilated remodeled ventricle after anterior myocardial infarction. RESTORE group. Reconstructive Endoventricular Surgery, returning Torsion Original Radius Elliptical Shape to the LV. J Am Coll Cardiol. 2001;37:1199-209.

11. Starling RC, McCarthy PM, Buda T, Wong J, Goormastic M, Smedira NG, et al. Results of partial left ventriculectomy for dilated cardiomyopathy: hemodynamic, clinical and echocardiographic observations. J Am Coll Cardiol. 2000;36:2098-103.

12. Franco-Cereceda A, McCarthy PM, Blackstone EH, Hoercher KJ, White JA, Young JB, et al. Partial left ventriculectomy for dilated cardiomyopathy: is this an alternative to transplantation? J Thorac Cardiovasc Surg. 2001;121:879-93.

13. Dickstein ML, Spotnitz HM, Rose EA, Burkhoff D. Heart reduction surgery: an analysis of the impact on cardiac function. J Thorac Cardiovasc Surg. 1997; 113:1032-40.

14. Artrip JH, Oz M, Burkhoff D. Left ventricular volume reduction surgery for heart failure: a physiologic perspective. J Thorac Cardiovasc Surg. 2001;122:775-82.

15. Gorcsan J, Feldman AM, Kormos RL, Mandarino WA, Demetris AJ, Batista RJV. Heterogeneous immediate effects of partial left ventriculectomy on cardiac performance. Circulation. 1998;97:839-42.

16. Tulner SA, Steendijk P, Klautz RJ, Bax JJ, Schalij MJ, van der Wall $\mathrm{EE}$, et al. Surgical ventricular restoration in patients with ischemic dilated cardiomyopathy: evaluation of systolic and diastolic ventricular function, wall stress, dyssynchrony, and mechanical efficiency by pressure-volume loops. J Thorac Cardiovasc Surg. 2006;132:610-20.

17. Burkhoff D, Mirsky I, Suga H. Assessment of systolic and diastolic ventricular properties via pressure-volume analysis: a guide for clinical, translational, and basic researchers. Am J Physiol Heart Circ Physiol. 2005;289:H501-12.

18. Baan J, van der Velde ET, DeBruin HG, Smeenk GJ, Koops J, Van Dijk $\mathrm{AD}$, et al. Continuous measurement of left ventricular volume in animals and humans by conductance catheter. Circulation. 1984;70:812-23.

19. Zile MR, Brutsaert DL. New concepts in diastolic dysfunction and diastolic heart failure: Part I: diagnosis, prognosis, and measurements of diastolic function. Circulation. 2002;105:1387-93.

20. Lazar HL, Haasler GB, Collins RH, Dubroff JM, Meisner J, Spotnitz HM. Mechanisms of altered ventricular compliance following ischemia using two-dimensional echocardiography. Curr Surg. 1982;39:253-5.

21. Dor V, Sabatier M, Di Donato M, Montiglio F, Toso A, Maioli M. Efficacy of endoventricular patch plasty in large postinfarction akinetic scar and severe left ventricular dysfunction: comparison with a series of large dyskinetic scars. J Thorac Cardiovac Surg. 1998;116:50-9.

22. Schreuder JJ, Castiglioni A, Maisano F, Steendijk P, Donelli A, Baan $\mathrm{J}$, et al. Acute decrease of left ventricular mechanical dyssynchrony and improvement of contractile state and energy efficiency after left ventricular restoration. J Thorac Cardiovasc Surg. 2005;129:138-45.

23. Athanasuleas CL, Buckberg GD, Stanley AW, Siler W, Dor V, Di Donato $\mathrm{M}$, et al. Surgical ventricular restoration in the treatment of congestive heart failure due to post-infarction ventricular dilation. J Am Coll Cardiol. 2004;44:1439-45. 